\title{
Immunomodulation by interference with co-stimulatory molecules: therapeutic perspectives in asthma
}

\author{
Samuele E Burastero, Giovanni A Rossi
}

In allergic asthma, allergen specific $\mathrm{T}$ lymphocytes of the CD4 subset ( $\mathrm{T}$ helper or Th cells) control the cellular and molecular events underlying the establishment and chronic maintenance of airway inflammation that characterises the disease. The basic immunological event is the shift of allergen reactive CD4 Th lymphocytes towards a Th2 phenotype with the production of Th 2 cytokines such as interleukin (IL)-4, IL-13, and IL-5. Th2 cells can support both allergen specific IgE production and eosinophil recruitment.

Recognition of allergen via the $\mathrm{T}$ cell receptor $(\mathrm{TcR})$ provides the trigger for a specific $\mathrm{T}$ cell response; as any antigen, allergen is seen by Th cells as one of a few possible peptides derived from proteolytic processing. Immunogenic peptides are presented on major histocompatibility complex (MHC) molecules expressed on the membrane of antigen presenting cells. However, antigen recognition can follow such opposite events as anergy or activation of $\mathrm{T}$ cells - that is, induction of tolerance versus establishment of an effective immune response. Current concepts recognise that the outcome of antigen recognition is dependent, at least in part, on the complex interaction between MHC molecule associated membrane proteins (collectively known as co-stimulatory molecules) on the antigen presenting cells and their ligands on the $\mathrm{T}$ cells. Moreover, when the immune response is activated, costimulation is also involved in determining which effector profile will be acquired by the $\mathrm{T}$ cells - that is, a Th1 or a Th2 phenotype.

We will briefly review the experimental evidence supporting the concept that interference with the proper co-stimulatory molecules may allow the design of pharmaceutical products which cause immune deviation that could quantitatively and qualitatively change the $\mathrm{T}$ cell response. This approach could interfere with the chronic amplification of allergic inflammation at the level of the target organ in atopic individuals. 20132 Milan, Italy

S E Burastero

G Gaslini Institute, Genoa, Italy

G A Rossi

Correspondence to: Dr S E Burastero.

Received 20 November 1998 Returned to authors 8 December 1998

Revised manuscript received

19 January 1999

Accepted for publication

26 January 1999

\section{Co-stimulatory molecules and antigen presentation}

Alveolar macrophages and dendritic cells are resident phagocytes in the lung. Physiologically, alveolar macrophages act as very poor antigen presenting cells and, indeed, they are considered as inhibitors of local $\mathrm{T}$ cell activation. ${ }^{1}$ This characteristic of alveolar macrophages is thought to prevent the inappropriate activation of the lung immune system, which is continuously exposed to inert airborne antigens, and thus prevent a persistent inflammatory reaction which could lead to tissue damage and impairment of gas exchange. ${ }^{2}$

The poor antigen presenting ability of alveolar macrophages in healthy individuals may be related to reduced expression and/or function of membrane co-stimulatory molecules on the cell surface. ${ }^{3}$ On the other hand, the increased antigen presenting function of alveolar macrophages found in chronic inflammatory disorders such as allergic asthma and pulmonary sarcoidosis $^{4}$ may be a result of the enhanced expression of co-stimulatory molecules by these cells. Indeed, in different murine models of asthma the key role of the expression of two co-stimulatory molecules (CD80 (or B7.1) and CD86 (B7.2)) on alveolar macrophages in the maintenance of allergic inflammation has been progressively elucidated.

In contrast to alveolar macrophages, dendritic cells constitutively express costimulatory molecules such as the B7 molecules CD80 and CD86. Dendritic cells are located in the interstitial lung tissue ${ }^{5}$ and act as highly efficient antigen presenting cells carrying inhaled particles with antigenic properties (usually from pathogens) to the regional lymph nodes. ${ }^{6}$ In these organised immune sites naive $T$ cells bearing the relevant specificities are located. In response to antigen presentation, allergen specific $T$ and $B$ cells are expanded. In this fashion they acquire the phenotype of "memory" lymphocytes with recirculation capabilities (dependent on a defined array of homing receptors ${ }^{7}$ ) allowing them to reach the lung epithelium and activate their effector function at this site.

The presence of CD80 and CD86 molecules on antigen presenting cells provides a major co-stimulatory signal for activation of $\mathrm{T}$ cells through the $\mathrm{T}$ cell counter receptor, the CD28 molecule (fig 1). ${ }^{8}$ Antigen recognition, in the absence of CD28 co-stimulation, results in lack of IL-2 mRNA induction ${ }^{9}$ and in $\mathrm{T}$ cell anergy. ${ }^{10}$ In contrast, in the presence of CD28 co-stimulation, antigen recognition results in IL-2 mRNA stabilisation and accumulation and in $\mathrm{T}$ cell activation. ${ }^{11}$

Upon activation an additional ligand of CD80 and CD86, the CTLA-4 molecule, is expressed by T cells. ${ }^{12}{ }^{13}$ CTLA-4 binds the B7 molecules CD80 and CD86 with 20-50 times higher affinity than does CD28 but, in contrast to CD28, delivers downregulatory signals (table 1). ${ }^{13}$

The B7 molecules are expressed by antigen presenting cells in a fashion which depends on the type and on the state of activation of the cells. Indeed, blood monocytes constitutively express CD86, while CD80 is induced only 

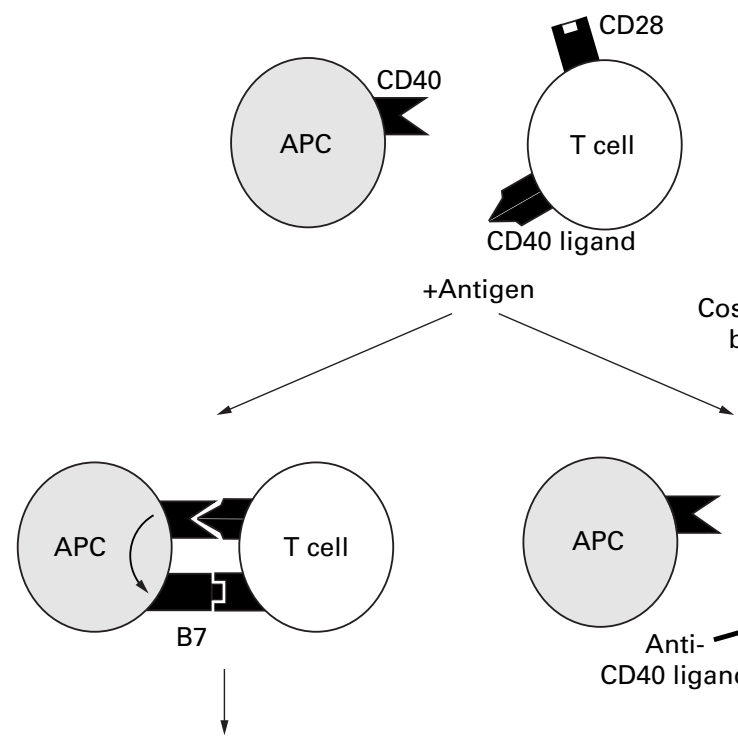

Anti-

Unresponsiveness

Active immune response

ostimulation

blockade
CD40 ligand

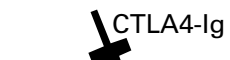

Figure 1 Antigen presenting cells express the CD40 molecule (alveolar macrophages from patients with sarcoidosis express this molecule while healthy controls do not). The corresponding ligand (CD40 ligand or CD153) is constitutively expressed on lymphocytes, but its expression is upregulated on encounter with an antigen. These events further upregulate expression of $B 7$ molecules on antigen presenting cells, thus allowing the engagement of the CD28 molecule on T cells. At this point, an effective antigen specific immune response can develop. Molecules which interfere with one or both co-stimulatory pathways may be promising candidates as innovative anti-inflammatory drugs.

upon activation of these cells. In contrast, dendritic cells, which are more potent antigen presenting cells, constitutively express both CD80 and CD86.

Antigen presentation by non-professional antigen presenting cells on normal healthy tissues are, in principle, either tolerance inducing or do not support the maturation of the immune response because of the absence of co-stimulatory molecules. Indeed, while the expression of MHC molecules on activated endothelium ${ }^{14}{ }^{15}$ and epithelium ${ }^{16}{ }^{17}$ is regulated by several stimuli, usually this is not associated with the expression of B7 co-stimulatory molecules. However, in some circumstances other membrane molecules can serve this functionfor example, LFA-3 ${ }^{18}$ or ICAM-1. ${ }^{19}$ Notably, human Th1 and Th2 clones were reported not to be susceptible to inhibition by CD80 or CD86. ${ }^{20}$ Moreover, Yi-qun et al reported that, in vitro, activated $T$ cells could be restimulated in the absence of CD80 or CD86 co-stimulation. ${ }^{21}$ It may therefore be possible that the expression of class II MHC molecules in vivo by non-professional antigen presenting cells could support the proliferation of defined lymphocyte subsets in peripheral tissues. It is likely that these complex interactions differentially contribute to the establishment and

Table 1 Co-stimulatory molecules and their ligands

\begin{tabular}{lll}
\hline Alveolar macrophages & Tlymphocytes & \\
\hline CD40 & CD154 or CD40 ligand & \\
CD80 (B7.1) & CD28 & CTLA4 $^{3}$ \\
CD86 (B7.2) & CD28 & CTLA4 $^{3}$ \\
\hline
\end{tabular}

${ }^{1}$ Expressed upon $\mathrm{T}$ cell receptor $(\mathrm{TcR})$ engagement by antigen (i.e. signal 1).

${ }^{2}$ Constitutively expressed.

${ }^{3}$ Expressed upon activation via signal 1 , modulated via signal 2 (e.g. B7/CD28 dependent co-stimulation). amplification of several inflammatory reactions in single target organs, depending on the phenotype of the $T$ cells on the one hand and the type and state of activation of antigen presenting cells on the other.

The expression of the B7 molecules may also be upregulated by the interaction between other co-stimulatory molecules expressed by antigen presenting cells and $\mathrm{T}$ cells such as the CD40-CD40 ligand or CD154. ${ }^{22}$ This pathway, initially described as having a role in B cell activation, has been recognised as having a key role for $\mathrm{T}$ cell activation also. ${ }^{22} \mathrm{CD} 40$ is expressed on antigen presenting cells such as macrophages, dendritic cells, and B cells, as well as by other cell types such as endothelial cells. $^{23}$ The ligand for CD40 not only signals positively for antibody production by $\mathrm{B}$ cells, but also induces strongly the expression of B7 molecules $^{24} 25$ and the secretion of inflammatory cytokines that participate in $\mathrm{T}$ cell activation. $^{26}$

\section{Lessons on the role of CD80 and CD86 in immunity and tolerance from knock out mice}

Mice genetically deficient in CD80 or CD86 and double knock out mice have recently been generated. ${ }^{27} 28$ Mice lacking either CD80 or CD86 remained capable of generating $T$ cell dependent humoral immune responses, suggesting that the co-stimulatory functions of these molecules are redundant. However, in the same studies CD86 knock out mice were less responsive to immunisation than CD80 knock out mice, suggesting a primary role for CD86 in the initiation of immune responses. Furthermore, both $\mathrm{Th} 1$ and $\mathrm{Th} 2$ responses were reduced in CD86 knock out mice, indicating that immunodepression is not associated with immunodeviation. Finally, CD80/ CD86 double knock out mice showed the most severe impairment in their immunity, supporting the view that CD80 and CD86 act cooperatively to modulate $\mathrm{T}$ cell activation. Recently, Schweitzer et al reported that induction of IL- 4 production and Th2 differentiation by naive T cells is highly dependent on B7 molecules, whereas IL-4 production by previously activated $\mathrm{T}$ cells is not. ${ }^{29}$ These authors found that the predominant contribution of B7 mediated signals to Th1 cytokine production by both naive and primed T cells was on IL-2 production (and expansion) rather than on IFN- $\gamma$ (effector cytokine) production. They therefore concluded that the antigenic experience of a $\mathrm{T}$ cell at the time of B7 blockade may determine whether blockade predominantly affects $\mathrm{T}$ cell expansion, differentiation, or effector cytokine production. These results indicate the variables that need special attention in evaluating the effect of co-stimulation blockade in any given experimental system. It will be interesting to evaluate the characteristics of experimentally induced asthma in B7 knock out mice to explore different functional roles for CD80 and CD86 in these models (see below). 
Involvement of CD80 and CD86 in allergic asthma

The CD80 co-stimulatory molecule is abnormally expressed on alveolar macrophages, both at baseline and after allergen challenge, in atopic individuals with asthma. ${ }^{30}{ }^{31}$ There were no differences in the alveolar macrophage expression of CD86 between healthy controls and allergic asthmatic subjects, both at baseline and after allergen challenge. In in vitro experiments to characterise the functional relevance of this finding, we observed that the secretion of IL-4 and IL-5 by autologous allergen specific Th2 cells, as well as their in vitro proliferative response to allergen, were all highly dependent on CD80. ${ }^{32}$ Interestingly, in allergic asthma the proportion of alveolar macrophages expressing CD80 was highly correlated with eosinophil recruitment to the lung induced by allergen inhalation challenge, further stressing the relevance of this co-stimulatory molecule in the maintenance of inflammation in the allergic lung. It is likely that the presence of the molecular prerequisites for an efficient antigen presenting function can explain the expansion of allergen specific Th2 CD4 cells in the lower respiratory tract of allergic asthmatic subjects ${ }^{32}$ but not in non-atopic controls. ${ }^{33}$

Larché et $a l^{34}$ have recently reported that co-stimulation through CD86 is involved in the expansion of $\mathrm{T}$ cell responses to allergen in atopic asthmatic subjects; they found that both allergen induced proliferation and IL-5 secretion were inhibited by CTLA4-Ig fusion protein and anti-CD86 (but not by anti-CD80) monoclonal antibodies. In particular, they reported that IL-5 production is inhibited by CD86 blockade in a manner independent of IL-2 since exogenously added IL-2 could restore $\mathrm{T}$ cell proliferation but not IL-5 production. Moreover, they found that $\mathrm{T}$ cells from bronchoalveolar lavage fluid can proliferate on addition of allergen in a CD86 dependent fashion. However, these authors could not formally conclude that CD80 contributed to the activation of allergen specific $T$ cells in asthmatic subjects in defined steps of initiation, maintenance, and amplification of the allergic immune response. Although the mechanisms of local amplification of the immune response may be additional to the recruitment of lymphocytes from peripheral blood, ${ }^{35}{ }^{36}$ these results suggest that co-stimulation treatment targeted at $\mathrm{T}$ cells could be of considerable interest in allergic asthma.

\section{Therapeutic potential of co-stimulation blockade}

A recombinant fusion protein consisting of the extracellular domain of CTLA-4 linked to the constant region of $\operatorname{IgG}_{1}$, known as CTLA4-Ig, binds $\mathrm{B} 7$ molecules with an affinity similar to membrane CTLA-4. It therefore acts as a powerful inhibitor of $\mathrm{B} 7 / \mathrm{CD} 28$ mediated co-stimulation (fig 1). ${ }^{37}$ Interestingly, in murine models of allergic asthma CTLA4-Ig had a powerful inhibitory effect on the allergic inflammation and its functional consequences. ${ }^{38} 39$ This protein could therefore represent a powerful "immunomodulator" with the potential to suppress an unwanted ongoing immune response to allergens in humans.

Lack of systemic effect is expected since this molecule has also been produced as a chimera consisting of the human IgG constant region engineered with the extracytoplasmic domain of human CTLA-4. Moreover, CTLA4-Ig did not produce significant side effects in large animal models when administered systemically. ${ }^{40}$ It is tempting to speculate that its use by inhalation could offer further advantages in terms of flexibility, incidence of side effects, and cost.

A further possible method of interfering with co-stimulation is offered by CD40 ligand antagonists. Blocking the CD40/CD40 ligand co-stimulatory pathway by means of a monoclonal antibody against murine CD40 is effective in preventing allograft rejection in mice. ${ }^{41}$ It remains to be tested in animal models whether this approach may be relevant in controlling the allergic immune response. Moreover, the combined administration of CTLA4-Ig and anti-CD40 ligand could be even more effective, as suggested by recent reports in renal allograft models in mice ${ }^{41}$ and in non-human primates. ${ }^{42}$

It is still controversial whether, and to what extent, CD80 and CD86 provide qualitatively different signals to $\mathrm{T}$ lymphocytes which are progressing towards a given differentiation pathway (Th1 or Th2). For instance, CD80 and CD86 have distinct regulatory functions in animal models of Th1 autoimmune diseases. ${ }^{43-45}$ In murine models of allergy and asthma, independently set up by different groups, distinct roles have been attributed to CD80 and CD86 with regard to formal gain and loss of function. ${ }^{46-48}$ The results have been contradictory; Keane-Myers and Harris reported a dependence on CD86 in the establishment of allergic inflammation while Tsuyuki and collaborators concluded that CD80 was primarily involved in their animal model.

Attempts to obtain CD80 and CD86 specific ligands have been successfully carried out. Morton et al reported that five single amino acid substitutions within the CTLA-4 MYPPPY domain, while exerting a modest effect on CD80, completely abrogated CD86 binding. ${ }^{49}$ Moreover, substitutions in the $\mathrm{N}$-terminal of the MYPPPY region and within the CDR1-like region of CTLA-4 eliminated both CD80 and CD86 binding. Hence, CD80 and CD86 have distinct binding sites on CTLA- $4 .{ }^{47}$

Harris et al explored the effect of a mutant version of CTLA4-Ig in a mouse model of allergy and asthma and found that CD80, but not CD86, was necessary for the maintenance or amplification of lung inflammatory responses. ${ }^{47}$ Thus, the availability of molecules capable of interfering selectively with key co-stimulatory receptors on the membrane of alveolar macrophages might represent a relevant improvement in the search for refined immunomodulators. 


\section{Conclusions}

In allergic asthma the enhanced co-stimulatory activity exerted by alveolar macrophages to resident lung $\mathrm{T}$ lymphocytes is probably involved in the establishment and maintenance of chronic inflammation in the lower respiratory tract. Specific molecular tools capable of interfering with this upregulated function might allow the design of a new category of anti-inflammatory drugs in the fight against the immunological basis of inflammation in asthma.

This work was supported by a grant from Istituto Superiore di Sanità to SEB.

1 Hance AJ. Accessory cell-lymphocyte interactions. In: Crystal RG, Weibel ER, Barnes PJ, eds. The Lung: Scientific Foundations. 2nd ed. Philadelphia: Lippincott-Raven, 1997: 821-39.

2 Reynolds HY. Immunologic system in the respiratory tract. Physiol Rev 1991;71:1117-33.

3 Chelen CJ, Fang Y, Freeman GJ, et al. Human alveolar macrophages present antigen ineffectively due to defective rophages present antigen ineffectively due to defective
expression of B7 costimulatory cell surface molecules. expression of B7 costimulatory

4 Nicod LP, Isler P. Alveolar macrophages in sarcoidosis coexpress high levels of CD86 (B7.2), CD40, and CD30L. Am F Respir Cell Mol Biol 1997;17:91-6.

5 Schon-Hegrad MA, Oliver J, McMenamin PG, et al. Studies on the density, distribution, and surface phenotype of intraepithelial class II major histocompatibility complex antigen (Ia)-bearing dendritic cells (DC) in the conducting airways. F Exp Med 1991;173:1345-56.

6 Xia W, Pinto CE, Kradin RL. The antigen-presenting activities of Ia+ dendritic cells shift dynamically from lung to lymph node after an airway challenge with soluble antigen. F Exp Med 1995;181:1275-83.

7 Burastero SE, Rossi GA, Crimi E. Selective differences in the expression of homing receptors of helper lymphocyte the expression of homing receptors of helper lymphoc

8 June CH, Bluestone JA, Nadler LM, et al. The B7 and June CH, Bluestone JA, Nadler LM, et al. The B7 and

9 Mueller DL, Jenkins MK. Molecular mechanisms underlying functional T-cell unresponsiveness. Curr Opin Immuno ing functional T-ce

10 Harding FA, McArthur JG, Gross JA, et al. CD28-mediated signalling co-stimulates murine $\mathrm{T}$ cells and prevents induction of anergy in T-cell clones. Nature 1992;356:607-9.

11 Lindstein T, June CH, Ledbetter JA, et al. Regulation of lymphokine messenger RNA stability by a surfacemediated T cell activation pathway. Science 1989;244:33943.

12 Lindsten T, Lee KP, Harris ES, et al. Characterization of CTLA-4 structure and expression on human T cells. $\mathcal{F}$ Immunol 1993;151:3489-99.

13 Tivol EA, Borriello F, Schweitzer AN, et al. Loss of CTLA-4 leads to massive lymphoproliferation and fatal multiorgan tissue destruction, revealing a critical negative regulatory role of CTLA-4. Immunity 1995;3:541-7.

14 Lian RH, Kotwal GJ, Wellhausen SR, et al. IFN-gammainduced MHC class II gene expression is suppressed in induced MHC class II gene expression is suppressed in 864-73.

15 Korpelainen EI, Gamble JR, Smith WB, et al. Interferongamma upregulates interleukin-3 (IL-3) receptor expression in human endothelial cells and synergizes with IL-3 in stimulating major histocompatibility complex class II expression and cytokine production. Blood 1995;86:17682.

16 Albert SE, Strutz F, Shelton K, et al. Characterization of a cis-acting regulatory element which silences expression of the class II-A beta gene in epithelium. F Exp Med 1994;180:233-40.

17 Bland PW, Whiting CV. Differential control of major histocompatibility complex class II I-Ek alpha protein expression in the epithelium and in subsets of lamina propria antigen-presenting cells of the gut. Immunology 1993;79: 107-11.

18 Savage CO, Brooks CJ, Harcourt GC, et al. Human vascular endothelial cells process and present autoantigen to human endothelial cells process and present auto

19 Seino K, Azuma M, Bashuda H, et al. CD86 (B70/B7-2) on endothelial cells co-stimulates allogeneic CD4+ T cells. Int Immunol 1995;7:1331-7.

20 Bashian GG, Braun CM, Huang SK, et al. Differential regulation of human, antigen-specific Th1 and Th2 responses
by the B-7 homologues, CD 80 and CD 86. Am $\mathcal{F}$ Respir Cell by the B-7 homologues, CD8
Mol Biol 1997;17:235-42.

21 Yi-qun Z, Joost van Neerven RJ, Kasran A, et al. Differential requirements for co-stimulatory signals from B7 family members by resting versus recently activated memory $\mathrm{T}$ cells towards soluble recall antigens. Int Immunol 1996;8 37-44.

22 Durie FH, Foy TM, Masters SR, et al. The role of CD40 in the regulation of humoral and cell-mediated immunity. Immunol Today 1994;15:406-11.
23 Reul RM, Fang JC, Denton MD, et al. CD40 and CD40 ligand (CD154) are coexpressed on microvessels in vivo in human cardiac allograft rejection. Transplantation 1997;64 1765-74.

24 Hancock WW, Sayegh MH, Zheng XG, et al. Costimulatory function and expression of CD40 ligand, CD80, and CD86 in vascularized murine cardiac allograft rejection. Proc Natl Acad Sci USA 1996;93:13967-72.

25 Ranheim EA, Kipps TJ. Activated T cells induce expression of B7/BB1 on normat or through a CD40dependent signal. 7 Exp Med 1993;177:925-35.

26 Guo Y, Wu Y, Shinde S, et al. Identification of a costimulatory molecule rapidly induced by CD40L as CD $44 \mathrm{H} . \mathcal{F}$ Exp Med 1996;184:955-61.

27 Borriello F, Sethna MP, Boyd SD, et al. B7-1 and B7-2 have overlapping, critical roles in immunoglobulin class switching and germinal center formation. Immunity 1997;6:30313.

28 Schweitzer AN, Borriello F, Wong RC, et al. Role of costimulators in $\mathrm{T}$ cell differentiation: studies using antigen-presenting cells lacking expression of CD80 or CD86. f Immunol 1997;158:2713-22.

29 Schweitzer AN, Sharpe AH. Studies using antigenpresenting cells lacking expression of both B7-1 (CD80) and B7-2 (CD86) show distinct requirements for B7 molecules during priming versus restimulation of Th2 but not Th1 cytokine production. F Immunol 1998;161:2762-71.

30 Burastero SE, Magnani Z, Confetti C, et al. CD80 expression on alveolar macrophages and presence of airway eosinophils in asthma and in rhinitis before and after allergen challenge. Eur Respir f 1998;12(Suppl 28):112s.

31 Burastero SE, Magnani Z, Confetti C, et al. CD80 molecule expression by alveolar macrophages (AM) is enhanced in allergic asthma and it is involved in allergen presentation to allergen-specific T-cells. Eur Respir f 1998;12(Suppl 28): $159 \mathrm{~s}$.

32 Burastero SE, Crimi E, Balbo A, et al. Oligoclonality of lung $\mathrm{T}$ lymphocytes following exposure to allergen in asthma. $\mathcal{F}$ Immunol 1995;155:5836-46.

33 Burastero SE, Borgonovo B, Gaffi D, et al. The repertoire of T-lymphocytes recovered by bronchoalveolar lavage from healthy nonsmokers. Eur Respir F 1996;9:319-27.

34 Larché M, Till SJ, Haselden BM, et al. Costimulation through CD86 is involved in airway antigen-presenting cell and $\mathrm{T}$ cell responses to allergen in atopic asthmatics. 7 Immunol 1998;161:6375-82.

35 Burastero SE, Fenoglio D, Crimi E, et al. Frequency of allergen-specific $\mathrm{T}$ lymphocytes in blood and bronchial response to allergen in asthma. F Allergy Clin Immunol 1993;91:1075-81.

36 Borgonovo B, Casorati G, Frittoli E, et al. Recruitment of circulating allergen-specific $T$ lymphocytes to the lung on allergen challenge in asthma. F Allergy Clin Immunol 1997; $100 \cdot 669-78$.

37 Linsley PS, Brady W, Urnes M, et al. CTLA-4 is a second receptor for the B cell activation antigen B7. $\mathcal{F}$ Exp Med 1991;174:561-9.

38 Van Oosterhout AJ, Hofstra CL, Shields R, et al. Murine CTLA4-IgG treatment inhibits airway eosinophilia and hyperresponsiveness and attenuates IgE upregulation in a murine model of allergic asthma. Am $\mathcal{F}$ Respir Cell Mol Biol 1997; 17:386-92.

39 Krinzman SI, De Sanctis GT, Cernadas M, et al. Inhibition of $T$ cell costimulation abrogates airway hyperresponsiveness in a murine model. $\mathcal{F}$ Clin Invest 1996;98:2693-9.

40 Pearson TC, Routenber KL, Abrams J, et al. Prolongation of renal allograft survival with CTLA4Ig in the Rhesus macaque. 17th American Society of Transplant Physicians, Chicago, 1997: 85

41 Larsen CP, Elwood ET, Alexander DZ, et al. Long-term acceptance of skin and cardiac allografts after blocking CD40 and CD28 pathways. Nature 1996;381:434-8.

42 Kirk AD, Harlan DM, Armstrong NN, et al. CTLA4-Ig and anti-CD40 ligand prevent renal allograft rejection in primates. Proc Natl Acad Sci USA 1997;94:8789-94.

43 Thompson CB. Distinct roles for the costimulatory ligands $\mathrm{B} 7-1$ and $\mathrm{B} 7-2$ in $\mathrm{T}$ helper cell differentiation? Cell 1995;81:979-82.

44 Kuchroo VK, Das MP, Brown JA, et al. B7-1 and B7-2 costimulatory molecules activate differentially the Th1/Th2 developmental pathways: application to autoimmune disease therapy. Cell 1995;80:707-18.

45 Lenschow DJ, Ho SC, Sattar H, et al. Differential effects of anti-B7-1 and anti-B7-2 monoclonal antibody treatment on the development of diabetes in the nonobese diabetic mouse. F Exp Med 1995;181:1145-55.

46 Keane-Myers A, Gause WC, Linsley PS, et al. B7-CD28/ CTLA-4 costimulatory pathways are required for the development of $\mathrm{T}$ helper cell 2 -mediated allergic airway responses to inhaled antigens. F Immunol 1997;158:20429 .

47 Harris N, Peach R, Naemura J, et al. CD80 costimulation is essential for the induction of airway eosinophilia. $\mathcal{F}$ Exp Med 1997;185:177-82.

48 Tsuyuki S, Tsuyuki J, Einsle K, et al. Costimulation through B7-2 (CD86) is required for the induction of a lung mucosal $\mathrm{T}$ helper cell 2 (TH2) immune response and altered airway responsiveness. F Exp Med 1997;185:16719.

49 Morton PA, Fu XT, Stewart JA, et al. Differential effects of CTLA-4 substitutions on the binding of human CD80 (B7-1) and CD86 (B7-2). F Immunol 1996;156:1047-54. 